\title{
OBSERVATION OF LONGITUDINAL DIFFUSION AND COOLING DUE TO INTRA-BEAM SCATTERING AT THE FERMILAB RECYCLER RING*
}

\author{
M. Hü and S. Nagaitsev, FNAL, Batavia, IL 60134, U.S.A.
}

\section{Abstract}

The Fermilab Recycler Ring is a high-vacuum fixed energy antiproton storage ring with both stochastic (at present) and the future electron cooling systems. In this paper the technique for diffusion rate measurement, beam parameters and the analysis of data are presented, as well as the effect of intra-beam scattering on the operational considerations for the storage and cooling of the antiproton beam in the Recycler.

\section{INTRODUCTION}

The Fermilab Recycler is a $3.3-\mathrm{km} 8.9-\mathrm{GeV} / \mathrm{c}$ fixed momentum storage ring located in the Fermilab Main Injector tunnel [1]. Table 1 present relevant Recycler parameters. The Run II Luminosity Upgrade Plan requires the Recycler to play a key role as the repository of large stacks of antiprotons $\left(6 \times 10^{12}\right)$ with the appropriate phase space characteristics to be used in collider stores. Small-angle multiple intrabeam scattering (IBS) is the dominant heating mechanism, which determines the equilibrium emittance achievable in the Recycler.

Table 1: Recycler ring parameters

\begin{tabular}{|l|c|c|}
\hline Parameter & Value & Units \\
\hline Average $\beta$-function, $\beta_{\text {ave }}$ & 30 & $\mathrm{~m}$ \\
\hline Max. dispersion & 2 & $\mathrm{~m}$ \\
\hline Transition, $\gamma_{\mathrm{t}}$ & 20.7 & \\
\hline $\begin{array}{l}\text { Typ. transverse beam } \\
\text { emittances (n, 95\%), } \varepsilon_{\mathrm{n}}\end{array}$ & $3-7$ & $\pi \mathrm{mm}$-mrad \\
\hline Number of antiprotons & $\leq 6$ & $10^{12}$ \\
\hline
\end{tabular}

The longitudinal IBS heating in particular has been of concern [1] because of a small longitudinal emittance required for Tevatron collisions. Figure 1 shows the calculated longitudinal IBS diffusion rate. The IBS theory from Ref. [2] has been used to make these calculations. Also, the measured Recycler lattice functions have been used. One can notice from Fig. 1 (solid line) that the longitudinal heating vanishes for a certain rms momentum spread, $\delta p_{0}$, and becomes cooling above this momentum spread. For comparison, also shown in Fig. 1 is the heating (cooling) rate calculated in a smooth, round-beam, zero-dispersion approximation using the value of the average $\beta$-function, $\beta_{\text {ave }}$ from Table 1. It has been pointed out previously [1] that one can exploit this feature of the longitudinal diffusion rate to minimize the longitudinal emittance growth by compressing the bunch length, and thus increasing the

\footnotetext{
*Work supported by the Universities Research Assos., Inc., under contract DE-AC02-76CH03000 with the U.S. Dept. of Energy. \#martinhu@fnal.gov
}

momentum spread, until the diffusion rate vanishes. In the Recycler ring this is accomplished by employing a barrier-bucket rf system.[3] For a typical transverse emittance of 5-7 $\pi \mathrm{mm}$-mrad the point of vanishing longitudinal heating can not be reached without losing the "tail" particles at the momentum aperture. A practical solution has been to maintain the momentum spread as high as possible (around $4 \mathrm{MeV} / \mathrm{c}$ ), while preserving the beam lifetime. Figure 2 shows the value of the rms momentum spread, $\delta p_{0}$, for which the diffusion rate vanishes, as a function of the transverse beam emittance.

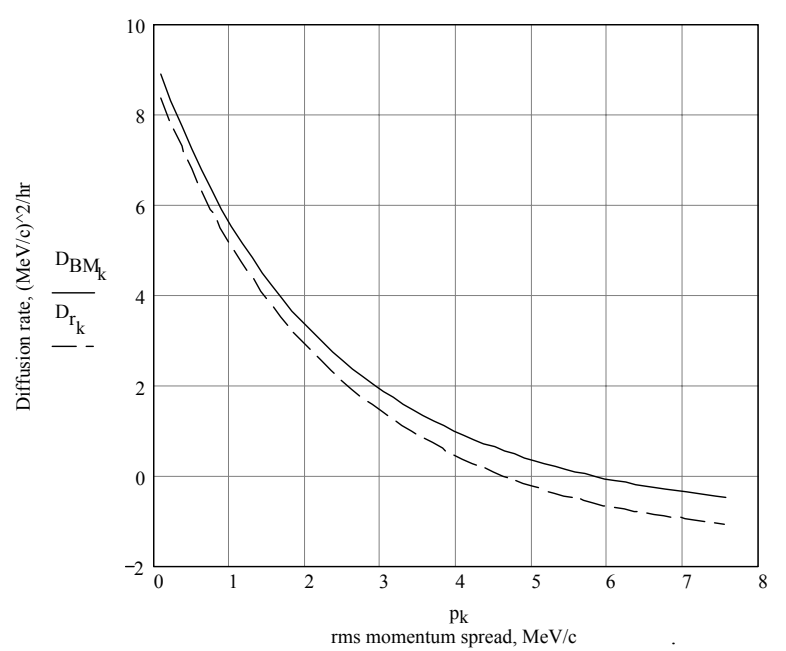

Figure 1: The calculated longitudinal IBS diffusion rate as a function of the rms beam momentum spread in the Recycler for a coasting beam of $100 \times 10^{12}$ antiprotons with a constant $5 \pi \mathrm{mm}$-mrad emittance. The solid line is modeled from Ref. [2]; the dashed line is the round-beam, zero-dispersion, smooth approximation.

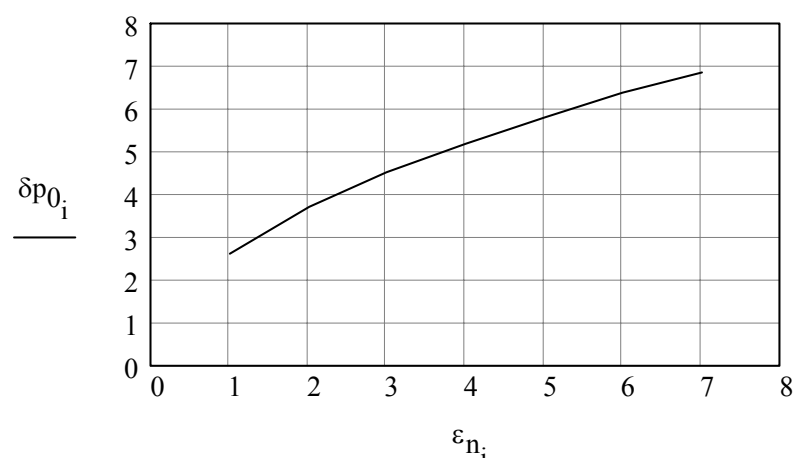

Figure 2: The value of the rms momentum spread in $\mathrm{MeV} / \mathrm{c}$, for which the longitudinal IBS heating vanishes, as a function of beam emittance (n, 95\%) in $\pi \mathrm{mm}$-mrad.

In the sections that follow we describe an experiment in which we created a beam distribution with the momentum spread less than $\delta p_{0}$ for a given emittance and observed 
longitudinal heating, and an experiment in which we created a beam distribution with the momentum spread greater than $\delta p_{0}$ for a given emittance and observed longitudinal cooling.

\section{LONGITUDINAL IBS HEATING}

To verify the IBS model we first created a beam distribution with a large transverse emittance and a small rms momentum spread. According to the IBS model this would correspond to longitudinal heating and small transverse cooling. The vacuum-related transverse heating rate was measured in a separate experiment by recording emittances of a coasting beam with very low current $\left(1 \times 10^{10}\right)$ to avoid the IBS contribution. This vacuum-related growth rate was measured to be $0.60 \pi \mathrm{mm}-\mathrm{mrad} / \mathrm{hr}$. Figure 3 shows the transverse emittance evolution for a costing beam of $100 \times 10^{10}$ protons.

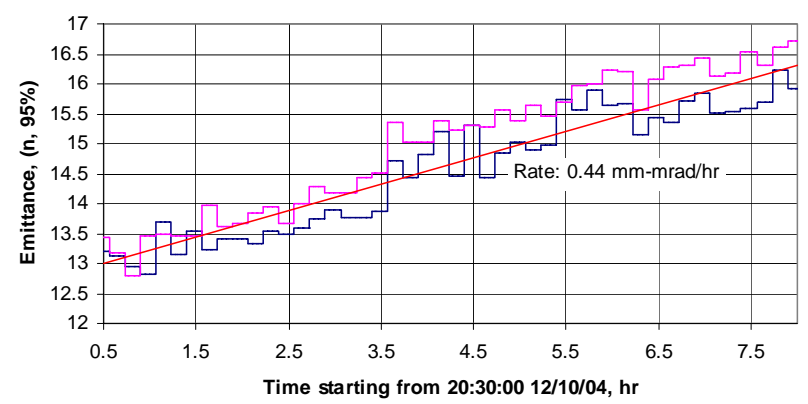

Figure 3: The transverse emittance (horizontal and vertical) evolution for a coasting beam of $100 \times 10^{10}$ protons in the Recycler. The linear fit yields a growth rate of $0.44 \pi \mathrm{mm}-\mathrm{mrad} / \mathrm{hr}$.

One can observe from Fig. 3 that the emittance growth rate is in fact smaller than that for a zero beam current. We attribute the difference to IBS-related transverse cooling, which, according to our model, is $-0.15 \pi \mathrm{mm}-$ $\mathrm{mrad} / \mathrm{hr}$.

The longitudinal degree of freedom allows us to observe the effect of IBS directly because it is the dominant effect. All other heating mechanisms are negligible for high beam currents. Figure 4 shows the evolution of the rms momentum spread for the same beam as in Fig. 3. The IBS model uses the transverse emittance growth rate as an input parameter.

\section{LONGITUDINAL IBS COOLING}

The second experiment was to demonstrate the longitudinal IBS cooling. The measurements were conducted with a bunched antiproton beam of $25 \times 10^{10}$, which was initially cooled transversely to a very small emittance $(<2 \pi \mathrm{mm}$-mrad). The momentum spread was increased to above $4.5 \mathrm{MeV} / \mathrm{c}$ by increasing the amplitude of the rf voltage and thus compressing the beam. Figures 5 and 6 show the measured transverse emittance and longitudinal rms momentum spread evolutions. Also shown is the IBS model. The only adjustable parameter in this model is the vacuum-related emittance growth rate. The best fit corresponds to this rate being $0.55 \pi \mathrm{mm}$ $\mathrm{mrad} / \mathrm{hr}$ - consistent with our previous measurements.

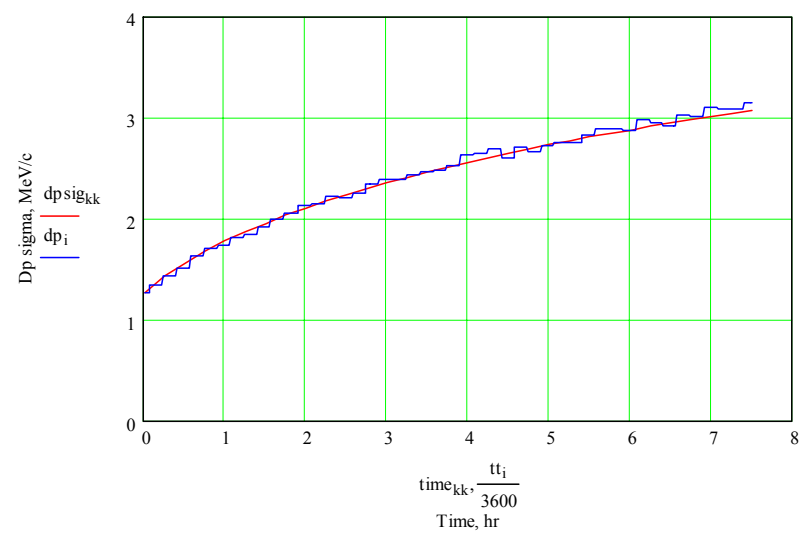

Figure 4: A comparison of the measured rms momentum spread $(\mathrm{MeV} / \mathrm{c})$ and the IBS model as a function of time for a coasting beam of $100 \times 10^{10}$ protons.

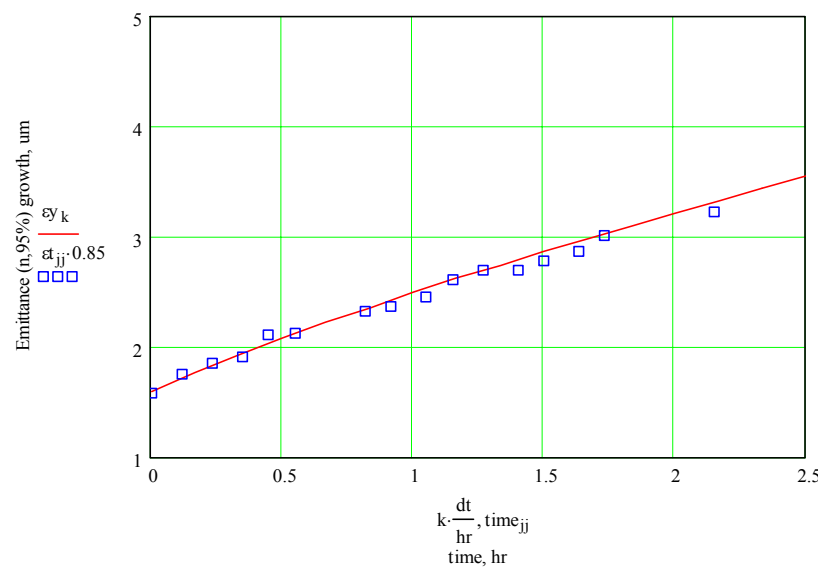

Figure 5: The transverse emittance evolution for a bunched antiproton beam $\left(25 \times 10^{10}\right)$.

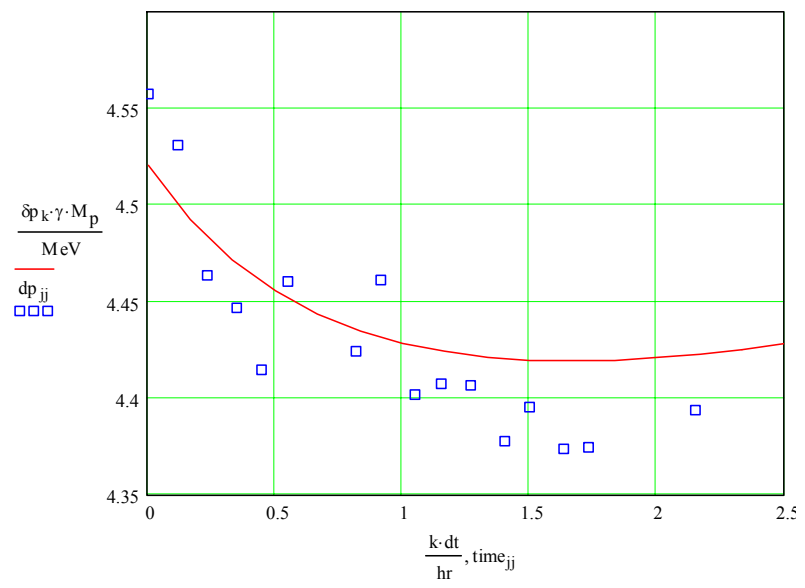

Figure 6: The longitudinal rms momentum spread $(\mathrm{MeV} / \mathrm{c})$ as a function of time (hours). The IBS model (solid line) has only one adjustable parameter - the vacuum-related transverse emittance growth rate. 


\section{IBS AND RECYCLER OPERATION}

At the current stage in Recycler commissioning, the Recycler has been operational in generating luminosity in the Tevatron since February, 2005. Figure 7 describes a typical scenario of cooling the antiproton beam after a transfer from the antiproton accumulator, prior to extraction to the Tevatron. Shown in this plot are beam intensity, beam lifetime, transverse emittance, rms momentum spread and longitudinal emittance over about 6 hours of beam storage. As soon as a nominal lifetime was reached through aggressive initial transverse cooling, the rms momentum spread was kept constant (around 4 $\mathrm{MeV} / \mathrm{c}$ ) by operationally compressing the bunch length with RF bucket manipulation. The slight rise at the end in the transverse emittance is expected as the transverse diffusion rate due to IBS increases as a function of the increasing longitudinal charge density. We have demonstrated that beam can be cooled optimally if we keep the rms momentum width large $(\sim 4 \mathrm{MeV} / \mathrm{c})$ while still maintaining good beam lifetime ( $\sim 500$ hours). Conversely, the longitudinal emittance of the beam never cools to this level if the above manipulation is not followed.

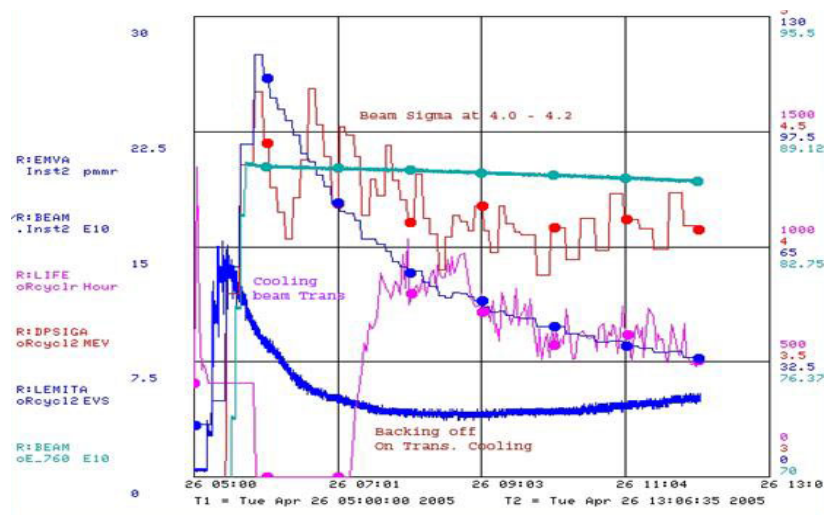

Figure 7: Demonstration of IBS-assisted longitudinal cooling: the rms momentum spread was kept at about 4 $\mathrm{MeV} / \mathrm{c}$ as the beam cooled longitudinally, so as to take advantage of small IBS rate.

\section{CONCLUSION}

We have developed a precise IBS model for the Recycler. This model has been verified with beam measurements made during storage and cooling of the antiprotons before extraction to the Tevatron. We have separately verified longitudinal IBS heating and cooling effects by designing the beam parameters to demonstrate the effects. Furthermore, the good agreement observed between model and measurement increased our confidence in the diagnostics. This IBS-assisted approach to beam cooling has been incorporated in our storage and cooling procedure for antiproton beam for luminosity generation.

\section{REFERENCES}

[1] Jackson, G., "The Fermilab Recycler Ring Technical Design Report,” Fermilab-TM-1991, Nov. 1996.

[2] J.D. Bjorken and S. Mtingwa, "Intrabeam Scattering", Particle Accelerators, 13, pp. 115-143 (1983).

[3] Lee, S.Y. and Ng, K.Y., "Particle dynamics in storage rings with barrier rf systems", Physical Review E, volume 5, number 5 (1997).

\section{ACKNOWLEDGEMENT}

We would like to thank Alexey Burov for his contribution into the formulation of the IBS model. 